\title{
Management of clozapine-resistant schizophrenia
}

\author{
Rob W. Kerwin \& Anusha Bolonna
}

\begin{abstract}
The incidence of treatment resistance in schizophrenia (failure to respond to antipsychotic therapy) is about $20 \%$. Factors that may contribute to it include non-adherence (non-compliance) to treatment, comorbid conditions and medication side-effects. The National Institute for Clinical Excellence recommends that clozapine be used for schizophrenia resistant to another atypical antipsychotic. Here we focus on patients who are also resistant to clozapine given in adequate dosage for sufficient duration. Switching from clozapine to a previously untried atypical (e.g. olanzapine, risperidone, quetiapine) might be of benefit in partial treatment resistance. In more difficult cases, augmentation of clozapine with benzamides (sulpiride, amisulpride) and anti-epileptics (lamotrigine) shows some success. In extreme treatment resistance, a strategy is recommended that combines the proven best drug for the particular patient and psychosocial treatments.
\end{abstract}

This article is the first of two in this issue in the series introduced by Robin McCreadie's editorial 'Schizophrenia revisited' (McCreadie, 2004). In the second, Connolly E Kelly (2005) discuss physical health and lifestyle. Previous contributions have considered environmental influences (Leask, 2004), implementation of the NICE schizophrenia guidelines (Rowlands, 2004), cognitive deficits in first-episode schizophrenia (Gopal $\mathcal{E}$ Variend, 2005) and early intervention in psychosis (Singh $\mathcal{E}$ Fisher, 2005).

In general, all patients with schizophrenia could be considered treatment resistant, since full remission is unusual in this disorder. In order to create specific treatment algorithms for patients that are more difficult to treat, it is necessary to define treatment resistance. Kane (1996) specified the following criteria for treatment resistance:

- available medications and other treatments are not useful in alleviating the target symptoms of schizophrenia (not only the positive and negative symptoms, but also disorganised or violent/aggressive behaviour, thought disorder and suicidal ideation);

- occurrence of adverse side-effects of medication;

- non-adherence to current treatment;

- presence of comorbid conditions such as substance misuse;

- failure of maintenance and relapse despite seemingly adequate doses of antipsychotics.

In addition, arbitrary defining criteria include failure to respond to at least two neuroleptic drugs equivalent to $600 \mathrm{mg}$ chlorpromazine per day for more than 4 weeks (Juarez-Reyes et al, 1995). In clinical trial and audit settings, the most frequently used criteria are those adopted by Kane et al (1988) for their seminal trial of clozapine $v$. chlorpromazine in treatment-refractory schizophrenia. In this trial, patients were classified as resistant to treatment if:

1 they had not demonstrated improvement after three periods of treatment with neuroleptics (from two or more different chemical classes) in the previous 5 years equivalent to $1000 \mathrm{mg}$ per day of chlorpromazine for 6 weeks;

2 patients had had no episodes of good functioning in the previous 5 years.

Using these criteria, Kane et al found the incidence of treatment resistance in schizophrenia to be $20 \%$.

\section{Management with clozapine}

This article focuses principally on the pharmacological management of treatment resistance. However, before initiating clozapine therapy, some basic principles should be adhered to, such as assessing non-adherence, re-evaluating the diagnosis, considering organic contributions to the diagnosis and assessing comorbidity (Morrison, 1996).

The evidence for clozapine as a superior treatment for schizophrenia is consistent and is the subject of a number of reviews and meta-analyses (Kane, 1992; Meltzer, 1992; Brambilla et al, 2002; Iqbal et al, 2003). Primary references for clozapine's superior efficacy

Rob W. Kerwin is Professor and Head of the Section of Clinical Neuropharmacology at the Institute of Psychiatry (De Crespigny Park, London SE5 8AF, UK. E-mail: r.kerwin@iop.kcl.ac.uk). His interests lie in all aspects of antipsychotic clinical psychopharmacology. Anusha Bolonna is an honorary research fellow at the Institute, with special interest in the genetics of drug response in schizophrenia. 
Table 1 Symptom domains of schizophrenia targeted by antipsychotics (after Pantelis \& Lambert, 2003)

\begin{tabular}{|c|c|c|}
\hline Symptom domain & Clinical features & Comments \\
\hline Positive & $\begin{array}{l}\text { Delusions, hallucinations, formal } \\
\text { thought disorder }\end{array}$ & $\begin{array}{l}\text { SGAs } \geq \text { FGAs. Clozapine has superior } \\
\text { efficacy in treatment-resistant patients }\end{array}$ \\
\hline Negative & $\begin{array}{l}\text { Avolition, apathy, anhedonia, } \\
\text { affective blunting, poverty of speech }\end{array}$ & $\begin{array}{l}\text { SGAs > FGAs. Few studies specifically examine } \\
\text { negative symptoms as primary outcome, but } \\
\text { exceptions are studies of amisulpride }\end{array}$ \\
\hline Cognitive & $\begin{array}{l}\text { Deficits in memory, attention, executive } \\
\text { function, judgement and insight }\end{array}$ & $\begin{array}{l}\text { SGAs > FGAs. Different SGAs may have } \\
\text { different effects on cognition }\end{array}$ \\
\hline Affective & $\begin{array}{l}\text { Altered stability of mood, manic-like } \\
\text { symptoms }\end{array}$ & $\begin{array}{l}\text { SGAs > FGAs. Some evidence that clozapine } \\
\text { and olanzapine are effective }\end{array}$ \\
\hline Suicidality & Suicidal ideation and behaviour & $\begin{array}{l}\text { Clozapine > olanzapine. No evidence available } \\
\text { for other SGAs and FGAs }\end{array}$ \\
\hline Behavioural & $\begin{array}{l}\text { Social withdrawal, antisocial behaviour, } \\
\text { incoherent and odd conversation }\end{array}$ & $\begin{array}{l}\text { Limited research available to assess drug } \\
\text { effects in this domain }\end{array}$ \\
\hline $\begin{array}{l}\text { Social and role } \\
\text { functioning, } \\
\text { quality of life }\end{array}$ & $\begin{array}{l}\text { Social interaction deficits, impaired } \\
\text { activities of daily living }\end{array}$ & $\begin{array}{l}\text { More research required. Some suggestion that } \\
\text { SGAs superior to FGAs }\end{array}$ \\
\hline
\end{tabular}

are cited in these reviews, but pivotal publications include a clinical trial of its use in treatment-resistant schizophrenia (Kane et al, 1988) and a meta-analysis of randomised controlled trials of clozapine (Wahlbeck et al, 1999).

Another further useful practical guide to the drug's use in treatment-resistant schizophrenia is a review by Pantelis \& Lambert (2003). In this, the authors suggest that treatment resistance is related to several symptom domains (Table 1) and they offer a threephase algorithm for the pharmacological management of incompletely recovered (treatment-resistant) patients (Fig. 1).

\section{Managing clozapine-resistant patients}

The most comprehensive review on this topic was published by Barnes et al (1996). Again, basic factors for clozapine-resistance highlighted in this review include comorbid drug misuse, poor adherence, inadequate duration of treatment and/or inadequate dosage. As Barnes et al note, two of these factors warrant further explanation.

The first is inadequate duration of treatment. It is accepted that a proportion of patients have a delayed response to clozapine (Meltzer, 1992). Meltzer concluded that $30 \%$ would respond by 6 weeks, a further $20 \%$ by 3 months and an additional $10-20 \%$ by 6 months. Therefore, it seems reasonable to try clozapine monotherapy for 6 months. This leaves a residue of $30 \%$ of patients for whom it must be decided whether to persevere with clozapine, consider various augmentation strategies or to cease clozapine therapy.

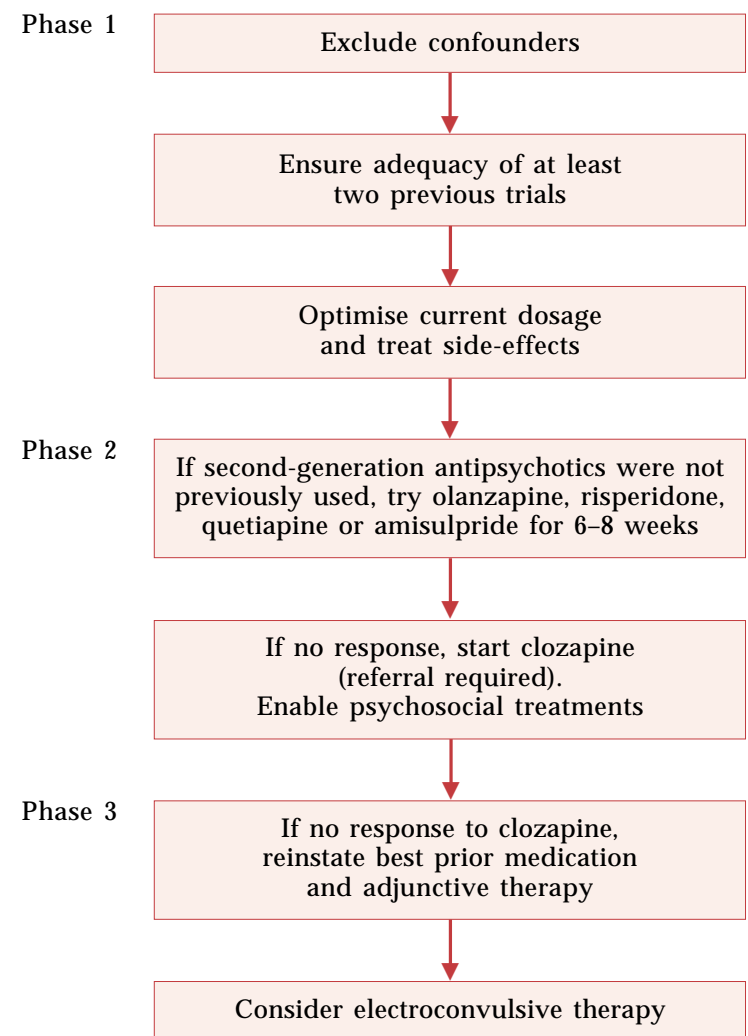

Fig. 1 Pharmacological management of incompletely recovered patients (after Pantelis \& Lambert, 2003). 
The second factor is inadequate dosage. Clozapine dosage can be a relatively complicated issue. In particular, there is no meaningful relationship between clozapine plasma levels and clinical response. However, there is a consensus in the literature that a plasma level of about 350-450 ng/ $\mathrm{ml}$ has to be attained before the patient is considered to be non-respondent to clozapine (Perry et al, 1991; Potkin et al, 1994).

Clozapine is also subject to considerable metabolism by the cytochrome P450 (CYP) enzyme system (Aitchison et al, 2000). There are numerous variants of the genes encoding the CYP enzyme family within the general population, resulting in complex individual genetic profiles and a variable response to drugs metabolised by these enzymes (Ma et al, 2004). ${ }^{+}$Therefore, in clinical practice, patients can be very susceptible to side-effects at drug dose levels that appear to be below the threshold for clinical efficacy.

\section{Augmentation of clozapine}

A frequent treatment strategy for clozapine-resistant patients with schizophrenia is the use of specific augmentors that are suitable for adjunctive therapy. Clozapine is a polyvalent drug but it lacks highpotency dopamine receptor blockade (Kerwin \& Osborne, 2000). Therefore, there has been interest in using as augmentors substituted benzamides with highly selective dopamine receptor blocking profiles (Kerwin, 2000). Augmentation strategies incorporating sulpiride are well documented. The authors of one study of sulpiride augmentation in 28 patients partially responsive to clozapine (Shiloh et al, 1997) noted a mean reduction of about $40-50 \%$ in various clinical response scores (Brief Psychiatric Rating Scale and Scale for the Assessment of Positive Symptoms).

Several groups have been interested in mimicking this study with amisulpride, a relative of sulpiride that is even more selective at the dopamine $D_{2}$ receptor. A case series by Zink et al (2004) showed improvement in previously treatment-resistant symptoms following a combined treatment strategy of clozapine and amisulpride. In addition, our group performed an open trial of amisulpride augmentation in a long-term (52 weeks) study. Significant improvement was observed in half of the patients, with no additional side-effects. Moreover, this study monitored plasma levels to determine whether this was a pharmacokinetic interaction.

${ }^{+}$The pharmacogenetics of clozapine treatment has been discussed in a previous issue of APT: see Tsapakis et al (2004). Ed.
Clozapine levels did not change throughout the duration of the trial, suggesting a pharmacodynamic interaction (Munro et al, 2004).

\section{Augmentation with anti-epileptics}

A glutamate hyperfunction hypothesis of schizophrenia has generated interest in the role of glutamate release inhibitors as clozapine augmentors. In a study of 26 treatment-resistant patients receiving lamotrigine (17) or topirimate (9) in addition to their existing antipsychotic treatment (a variety of antipsychotics), a significant improvement was observed when lamotrigine was added to risperidone, haloperidol, olanzapine or flupenthixol. However, no significant effect was observed in patients receiving topirimate augmentation in addition to clozapine, olanzapine, haloperidol or flupenthixol (Dursun \& Deakin, 2001). The therapeutic effects of lamotrigine augmentation were also assessed in a rigorous randomised placebo-controlled cross-over study of 34 clozapine-resistant patients (Tiihonen et al, 2003). In this 14-week study, lamotrigine treatment significantly improved positive symptoms and general psychopathological symptoms, but had no effect on negative symptoms. The authors suggested that this was the first time a non-dopamine antagonist had proven efficacy in schizophrenia, giving further credence to the hyperglutamate neurotransmission hypothesis for the generation of positive symptoms in the disorder.

\section{Are other atypicals, alone or in combination, of use?}

Other atypical antipsychotics were not trialled in the pre-registration phase for their efficacy in treatment resistance. However, there have been a number of phase IV trials that have examined this possibility.

\section{Risperidone}

The earliest study examining the possibility that risperidone may be of use in treatment resistance was conducted by Bondolfi et al (1998). This 8-week study of risperidone and clozapine concluded that the two drugs are comparable. However, the study has been criticised for its rapid titration schedule of 1 week, which may have led to clozapine intolerance and therefore to patients receiving suboptimal doses of the drug. Later studies include that by Wahlbeck et al (2000), which was a 10-week open-label trial of risperidone $v$. clozapine using a $20 \%$ decrease in Positive and Negative Symptom Scale score as the 
definition of clinical improvement. Intention-to-treat analysis did not identify significant differences between the two groups, but there was an unusually high dropout rate for clozapine (5 of 10 participants), seriously reducing the power of the study. To date, there has been no large, randomised, pragmatic trial of risperidone $v$. clozapine that has sufficient power to detect a difference between the treatments.

\section{Olanzapine}

The most informative study of olanzapine in treatment resistance is likely to be that by Conley et al (1998). This reproduced the methodology of the pivotal Kane et al (1988) study of clozapine but concluded that olanzapine was no better than chlorpromazine in treatment resistance. Additional studies using olanzapine following treatment failures with other antipsychotics (switching studies) have proved disappointing. However, one (Lindenmayer et al, 2002), although showing that olanzapine was not a useful switch drug for general psychopathological symptoms, did demonstrate an improvement in symptoms related to cognitive function. In a further switching study, Conley et al (1999) conducted 8-week open and double-blind trials administering olanzapine to 44 treatmentresistant patients and treating subsequent olanzapine-resistant patients with clozapine. Only $5 \%$ of patients responded to olanzapine and, of the remaining patients who were switched to clozapine, $41 \%$ responded to clozapine. The authors concluded that non-response to olanzapine does not predict failure to respond to clozapine. Some open studies (e.g. Dursun et al, 1999) suggest that olanzapine might be best used at higher doses in treatment-resistant schizophrenia.

\section{Quetiapine}

There is relatively little published information on controlled trials of quetiapine $v$. clozapine in treatment-resistant patients, but a number of small trials and case reports suggest its usefulness in treatment resistance (e.g. Fabre et al, 1995; Brooks, 2001).

\section{Non-clozapine atypicals in combination}

At the end of the algorithm shown in Fig. 1, many clinicians try ad hoc combinations of atypical antipsychotics for treatment failures with clozapine. This usually involves a combination of olanzapine and risperidone. There is a limited published evidence base on this strategy. Most publications in this area are case reports and there are likely to be very many unpublished cases where this strategy has been used in clinical practice. However, Lerner et al (2004) have attempted to review this scant literature. They found little evidence other than anecdotal case reports that augmentation of clozapine with other atypicals produces any benefit. Subtracting patients treated with combinations of clozapine and another atypical leaves a very small database on which to draw. However, Lerner et al concluded that there might be some benefit, which necessitates further controlled studies.

\section{Conclusions}

Although olanzapine and risperidone are probably the most successful antipsychotics for partially treatment-resistant patients, there is now very little controversy about the role of clozapine in more extreme treatment resistance. Indeed, the NICE schizophrenia guidelines of 2002 strongly recommend broader and earlier use of clozapine, suggesting that only one atypical should be tried before moving onto clozapine.

Most practitioners are now more concerned with managing clozapine-resistant patients, the focus of this short review. There is reasonable evidence to suggest that augmentation strategies with sulpiride, amisulpride and lamotrigine are useful in treatment resistance, but no indication as to which patient will benefit from which strategy. The small proportion of patients who remain densely resistant to any pharmacological treatment strategy should probably be managed by a historical review of their best treatment regime and the reinstatement of this along with psychosocial treatments.

\section{References and related articles}

Aitchison, K. J., Jann, M. W., Zhao, J. H., et al (2000) Clozapine pharmacokinetics and pharmacodynamics studied with Cyp1A2-null mice. Journal of Psychopharmacology, 14, 353-359.

Barnes, T. R. E., McEvedy, C. J. B. \& Nelson, H. E. (1996) Management of treatment resistant schizophrenia unresponsive to clozapine. British Journal of Psychiatry, 169 (suppl. 31), 31-40.

Bondolfi, G., Dufour, H., Patris, M., et al (1998) Risperidone versus clozapine in treatment-resistant chronic schizophrenia: a randomized double-blind study. The Risperidone Study Group. American Journal of Psychiatry, 155, 499-504.

Brambilla, P., Barale, F. \& Caverzasi, E. (2002) Clozapinetreated subjects with treatment-resistant schizophrenia: a systematic review of experimental and observational studies. International Journal of Clinical Psychopharmacology, 17, 189-195.

Brooks, J. O. III (2001) Successful outcome using quetiapine in a case of treatment resistant schizophrenia with assaultive behaviour. Schizophrenia Research, 50, 133-134.

Conley, R. R., Tamminga, C. A., Bartko, J. J., et al (1998) Olanzapine compared with chlorpromazine in treatmentresistant schizophrenia. American Journal of Psychiatry, 155, 914-920. 
Conley, R. R., Tamminga, C. A., Kelly, D. L., et al (1999) Treatment-resistant schizophrenic patients respond to clozapine after olanzapine non-response. Biological Psychiatry, 46, 73-77.

Connolly, M. \& Kelly, C. (2005) Lifestyle and physical health of people with schizophrenia. Advances in Psychiatric Treatment, 11, 125-132.

Dursun, S. M. \& Deakin, J. F. (2001) Augmenting antipsychotic treatment with lamotrigine or topiramate in patients with treatment-resistant schizophrenia: a naturalistic case-series outcome study. Journal of Psychopharmacology, 15, 297-301.

Dursun, S. M., Gardner, D. M., Bird, D. C., et al (1999) Olanzapine for patients with treatment-resistant schizophrenia: a naturalistic case-series outcome study. Canadian Journal of Psychiatry, 44, 701-704.

Fabre, L. F., Arvanitis, J., Pultz, V. M., et al (1995) ICI $204-$ 636 a novel atypical antipsychotic: early indications of safety and efficacy in patients with chronic and subchronic schizophrenia. Clinical Therapeutics, 17, 366-378.

Gopal, Y. V. \& Variend, H. (2005) First-episode schizophrenia: review of cognitive deficits and cognitive remediation. Advances in Psychiatric Treatment, 11, 38-44.

Iqbal, M. M., Rahman, A., Husain, Z., et al (2003) Clozapine: a clinical review of adverse effects and management. Annals of Clinical Psychiatry, 15, 33-48.

Juarez-Reyes, M. G., Shumway, M., Battle, C., et al (1995) Effects of stringent criteria on eligibility for clozapine among public mental health clients. Psychiatric Services, $46,801-806$

Kane, J. M. (1992) Clinical efficacy of clozapine in treatment refractory schizophrenia: an overview. British Journal of Psychiatry, 160 (suppl. 17), 41-45.

Kane, J. M. (1996) Factors which make patients difficult to treat. British Journal of Psychiatry, 169 (suppl. 31), 10-14.

Kane, J., Honigfeld, G., Singer, J., et al (1988) Clozapine for the treatment-resistant schizophrenic. A double-blind comparison with chlorpromazine. Archives of General Psychiatry, 45, 789-796.

Kerwin, R. W. (2000) From pharmacological profiles to clinical outcomes. International Journal of Clinical Psychopharmacology, 15, S1-S4.

Kerwin, R. W. \& Osborne, S. (2000) Antipsychotic drugs. Medicine, 28, 23-25.

Leask, S. J. (2004) Environmental influences in schizophrenia: the known and the unknown. Advances in Psychiatric Treatment, 10, 323-330.

Lerner, V., Libov, I., Kotler, M., et al (2004) Combination of "atypical" antipsychotic medication in the management of treatment-resistant schizophrenia and schizoaffective disorder. Progress in Neuropsychopharmacology and Biological Psychiatry, 28, 89-98.

Lindenmayer, J. P., Czobor, P. \& Volavka, J. (2002) Olanzapine in refractory schizophrenia after failure of typical or atypical antipsychotic treatment: an open-label switch study. Journal of Clinical Psychiatry, 63, 931-935.

Ma, J. D., Nafziger, A. N. \& Bertino, J. S. Jr. (2004) Genetic polymorphisms of cytochrome P450 enzymes and the effect on interindividual, pharmacokinetic variability in extensive metabolizers. Journal of Clinical Pharmacology, 44, 447-456.

McCreadie, R. G. (2004) Editorial: Schizophrenia revisited. Advances in Psychiatric Treatment, 10, 321-322.

Meltzer, H. Y. (1992) Treatment of the neurolepticnonresponsive schizophrenic patient. Schizophrenia Bulletin, 18, 515-542.

Morrison, D. P. (1996) Management of treatment refractory schizophrenia. British Journal of Psychiatry, 169 (suppl. 31), 15-20.

Munro, J., Matthiasson, P., Osborne, S., et al (2004) Amisulpride augmentation of clozapine: an open nonrandomized study in patients with schizophrenia partially responsive to clozapine. Acta Psychiatrica Scandinavica, 110, 292-298.

National Institute for Clinical Excellence (2002) Clinical Guideline 1. Schizophrenia: Core Interventions in the
Treatment and Management of Schizophrenia in Primary and Secondary Care. London: NICE.

Pantelis, C. \& Lambert, T. J. (2003) Managing patients with "treatment-resistant" schizophrenia. Medical Journal of Australia, 178 (suppl.), S62-S66.

Perry, P. J., Miller, D., Arndt, S. V., et al (1991) Clozapine concentrations and norclozapine plasma concentrations and clinical response of treatment refractory schizophrenic patients. American Journal of Psychiatry, 148, 231235.

Potkin, S. G., Bera, R., Gulaskeram, B., et al (1994) Plasma clozapine concentrations predict clinical response in treatment-resistant schizophrenia. Journal of Clinical Psychiatry, 55 (suppl. B), 133-136.

Rowlands, P. (2004) The NICE schizophrenia guidelines: the challenge of implementation. Advances in Psychiatric Treatment, 10, 403-412.

Shiloh, R., Zemishlany, Z., Aizenberg, D., et al (1997) Sulpiride augmentation in people with schizophrenia partially responsive to clozapine. A double-blind, placebo-controlled study. British Journal of Psychiatry, 171, 569-573.

Singh, S. P. \& Fisher, H. L. (2005) Early intervention in psychosis: obstacles and opportunities. Advances in Psychiatric Treatment, 11, 71-78.

Tiihonen, J., Hallikainen, T., Ryynanen, O. P., et al (2003) Lamotrigine in treatment-resistant schizophrenia: a randomized placebo-controlled crossover trial. Biological Psychiatry, 54, 1241-1248.

Tsapakis, E., Basu, A. \& Aitchison, K. J. (2004) Clinical relevance of discoveries in psychopharmacogenetics. Advances in Psychiatric Treatment, 10, 455-465.

Wahlbeck, K., Cheine, M., Essali, A., et al (1999) Evidence of clozapine's effectiveness in schizophrenia: a systematic review and meta-analysis of randomized trials. American Journal of Psychiatry, 156, 990-999.

Wahlbeck, K., Cheine, M. \& Tuisku, K. (2000) Risperidone versus clozapine in treatment-resistant schizophrenia: a randomized pilot study. Progress in Neuropsychopharmacology and Biological Psychiatry, 24, 911-922.

Zink, M., Knopf, U., Henn, F. A., et al (2004) Combination of clozapine and amisulpride in treatment-resistant schizophrenia: case reports and review of the literature. Pharmacopsychiatry, 37, 26-31.

\section{MCQs}

1 The incidence of treatment-resistant schizophrenia is:

a $5 \%$

b $10 \%$

c $20 \%$

d $50 \%$

e $75 \%$.

2 The most commonly used categorical definition of treatment resistance is :

a prolonged hospitalisation

b less than a $20 \%$ improvement on the BPRS

c symptoms enduring for more than 2 years

d persistent negative symptoms

e persistent cognitive symptoms.

3 Definitions of treatment resistance might also include:

a non-adherence

b failure of maintenance

c comorbidity

d aggression

e negative symptoms. 
4 The following drugs show evidence that they are specific clozapine augmentors:

a aripiprazole

b ziprasidone

c lamotrigne

d zolepine

e amisulpride.
5 The following drugs alone or in combination may be useful for partial treatment resistance:

a haloperidol at low dose

$\mathrm{b}$ risperidone

c olanzapine

d risperidone and olanzapine

e aripiprazole.

\section{Clinical Evidence Mental Health (third edition)}

Royal College of Psychiatrists/BMJ Publishing Group

This book contains the mental health section extracted from Clinical Evidence, Issue 11 (BMJ Publishing Group), the international source of the best available evidence for effective health care. The book presents clear, concise summaries of the current state of the evidence on the prevention and treatment of mental health disorders derived, where possible, from randomised controlled trials and systematic reviews.

This third edition covers 15 different mental health disorders, 27 questions, and over 200 interventions.

- Based on the latest Clinical Evidence search and appraisal results.

- Includes evidence on the short- and long-term treatment of mental health disorders and their complications.

- Assesses drug treatments, non-drug treatments and psychological therapies.

- Uses explicit methodology for selecting which evidence to summarise.

- Categorises interventions according to whether they have been found to be effective or not.

- Presents key messages and structured summaries of filtered evidence.

- Presents the balance between benefits and harms of intervention.

August 2004, Paperback, 210pp, ISBN 190467116 0, Price $£ 14.00$

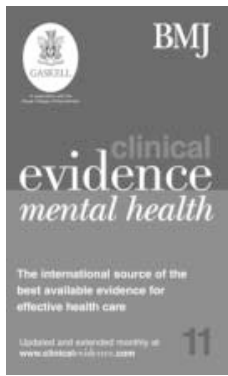

